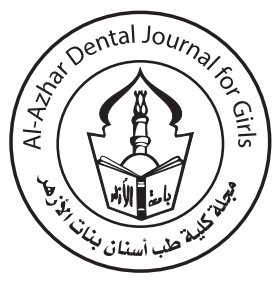

\title{
Evaluation of Resistin Levels in Gingival Crevicular fluid of Patients with Periodontitis and Type 2 Diabetes Mellitus after Non-Surgical Periodontal Therapy and Subantimicrobial Dosage of Doxycycline
}

\author{
Reem S. Abdu Shehab ${ }^{1^{*}}$, Eatemad A. Shoriebah ${ }^{2}$ and Mai Sh. Mansour ${ }^{3}$
}

Codex : 07/2001

azhardentj@azhar.edu.eg

http://adjg.journals.ekb.eg

DOI: $10.21608 /$ adjg.2019.7679.1089

\begin{abstract}
Purpose: This study was designed to evaluate the resistin levels in GCF of patients suffering from periodontitis and type 2 diabetes mellitus (T2DM) after non-surgical periodontal therapy and subantimicrobial dosage of doxycycline (SDD). Materials and methods: Thirty periodontal sites within 24 patients with age ranging between (24-51) years old were selected for this study. A total of 30 periodontal sites were randomly divided into three groups; each group included ten periodontal sites: (group I): systemically healthy periodontitis patients who had received scaling and root planning (SRP), (group II): periodontitis patients with T2DM who had received SRP, and (group III): periodontitis patients with T2DM who were received SRP and SDD 20mg twice/day. All patients were examined with the same clinical periodontal parameters which are plaque index (PI), gingival index (GI), probing depth (PD) and clinical attachment level (CAL). Patients in each group went through nonsurgical periodontal therapy as well as a maintenance program (which includes brushing with regular toothpaste). Collection of GCF samples was done in all patients at baseline, one month and 3 month following the periodontal therapy. Quantification of resistin in human samples was measured using resistin ELISA test. Results: Non-surgical periodontal therapy was found to show relative improvement in all clinical parameters as well as a decline in resistin levels. In addition, GCF levels of resistin, $\mathrm{PD}$, GI showed greater reduction after non-surgical treatment in group III than group II. Conclusion: Non-surgical periodontal therapy is
\end{abstract}

Resistin; Periodontitis;

Diabetes Mellitus; doxycycline

- Paper extracted from Master Thesis titled "Evaluation of Resistin Levels in Gingival Crevicular Fluid of Patients with Periodontitis and Type 2 Diabetes Mellitus after Non-Surgical Periodontal Therapy and Subantimicrobial Dosage of Doxycycline"

1. B.DS. 2010G Faculty of Dental Medicine University of Science and Technology -Sana'a-Yemen.

2. Professor of Oral Medicine, Periodontology, Diagnosis and Radiology department, Faculty of Dental Medicine for Girls, Al-Azhar University.

3. Associate Professor of Oral Medicine, Periodontology, Diagnosis and Radiology department, Faculty of Dental Medicine for Girls, Al-Azhar University.

* Corresponding author Email: reem.sh87@hotmail.com 
found to be effective in the management of periodontitis patients with and without diabetes mellitus and also GCF resistin can be a useful biomarker to detect the periodontal disease condition. Also, adjunctive use of SDD showed more clinical benefit to patients with periodontitis by host response modulation.

\section{INTRODUCTION}

Periodontitis can be defined as a chronic inflammatory disease that involves several factors. It is related to dysbiotic plaque biofilms in addition to escalating host-mediated destruction of the toothsupporting apparatus. It is mainly characterized by the loss of periodontal tissue support, seen clinically as an attachment loss (CAL) and radiographically assessed alveolar bone loss, existence of periodontal pocket and the bleeding of gingiva ${ }^{(1)}$.

Diabetes mellitus is known to be a major risk factor for periodontitis and maybe responsible for increased prevalence, severity and progression of periodontitis ${ }^{(2)}$. Many pathogenic mechanisms have been suggested and included alteration of oral microbial flora, vascular changes, abnormal defense mechanisms and altered collagen metabolism ${ }^{(3-4-5)}$. There has been much focus on the 'two-way' relationship between diabetes mellitus and periodontitis, which means that diabetes is not only a risk factor for periodontitis, but also periodontitis may affect glycaemic control negatively. The mechanisms that connect these conditions are not fully understood, but include aspects of immune functioning, activity of neutrophils as well as cytokine bi$\operatorname{olog} y^{(6)}$. Therefore, both periodontitis and diabetes mellitus follow similar pathogenic mechanism with inflammatory and pro-inflammatory cytokines such as TNF $\alpha$ IL-1 $\beta$, and IL- 6 that causing destruction in both conditions ${ }^{(7)}$. Also, it has been studied the association between adipokines, such as leptin, adiponectin and resistin, and inflammatory periodontal diseases in diabetic patients ${ }^{(5)}$.

Resistin is a recently discovered polypeptide secreted by adipocytes, that has been involved in the development of insulin resistance, that is why researchers named it as resistin (for resistance to insulin) ${ }^{(8)}$. It is also called adipocyte-specific secretory factor (ADSF), found in inflammatory zone (FIZZ), or resistin like molecules (RELM) ${ }^{(9)}$. The expression of resistin is detected in low levels in human adipose cells, and its abundance in peripheral blood mononuclear cells and macrophages. Also resestin acts as pro-inflammatory molecule that stimulating IL-12 and TNF- $\alpha$ synthesis ${ }^{(10)}$. It has been also demonstrated the resistin release is regulated from human neutrophils by Porphyromonas gingivalis lipopolysaccharide (P-LPS) which causes periodontitis ${ }^{(11)}$. So, resistin levels in gingival crevicular fluid (GCF) can be considered as a biomarker for studying the severity of periodontitis and/or periodontitis in diabetic patients ${ }^{(12)}$.

Several host modulation agents have been developed and are investigated nowadays such as NSAIDs, tetracyclines etc. All of these agents regulate specific constituents of disease pathogenesis which includes regulation of arachidonic acid metabolites, excessive production of matrix metalloproteinases (MMPs), immune and inflammatory responses and bone metabolism. Nevertheless, currently only one systemically administered agent, subantimicrobial dosage of doxycycline (SDD), has been approved by the United States FDA to be used for host modulation in periodontal diseaseas as an add-on to conventional nonsurgical periodontal therapy in the treatment of periodontitis ${ }^{(13)}$. SDD does not lead to antibiotic resistance by down-regulation of MMPs ${ }^{(14)}$.

Each patient's periodontal status was evaluated by measuring the plaque index $(\mathrm{Pl})^{(15)}$, gingival index $(\mathrm{GI})^{(16)}$, probing depth (PD), clinical attachment loss (CAL); at the baseline, one month and at 3 month intervals by using Williams graduated periodontal probe (with the following graduations: $1,2,3,4,5,6,7,8,9,10$ millimeters $)^{(17)}$.

Thus, the aim of the present study was to evaluate the resistin levels in GCF in patients with chron- 
ic periodontitis and T2DM after non-surgical periodontal therapy and SDD.

\section{SUBJECTS AND METHODS}

Thirty periodontal sites within 24 patients (19 females and 5 males) with age range (24-51) years old. Patients were selected from those attended to the Outpatient Clinics of Oral Medicine, Periodontology, Oral Diagnosis \& Radiology department, Faculty of Dental Medicine for Girls, AlAzhar University, clinically diagnosed as having at least one site with $\mathrm{CAL} \geq 5 \mathrm{~mm}$; moderate to advanced chronic periodontitis according to the criteria of American Academy of Periodontology ${ }^{[18]}$. The criteria for inclusion in the present study were including patients free from any systemic conditions that affect periodontium or interfere with periodontal treatment except type T2DM according to the modified Cornell Medical Index ${ }^{[19]}$, non-smoking, did not receive any periodontal treatment in the past six months before the examination, and not receive antibiotics or anti-inflammatory therapy in the six months before the examination, for female patients, no pregnancy or lactation was included.

All individuals were informed about the procedures of the study and benefits of their participation in the study. A satisfactory written consent was obtained from all the patients denoting they're convinced about the schedule research program design. Ethical committee meeting approved the study protocol.

Each patient's periodontal status was evaluated by measuring the plaque index $(\mathrm{Pl})^{(15)}$, gingival index $(\mathrm{GI})^{(16)}$, probing depth (PD), clinical attachment loss (CAL); at the baseline, one month and at 3 month ntervals by using Williams graduated periodontal probe (with the following graduations: 1,2,3,4,5,6,7,8,9,10 millimeters) ${ }^{(17)}$.The deepest PD was selected. Before the examination, the gingiva and the teeth were dried by a blast of air.

\section{Collection of Samples:}

Specimens of gingival crevicular fluid (GCF) were collected at the beginning, one month and three months following periodontal therapy. The samples were collected from the selected sites. The sample area was isolated with cotton rolls and cautiously cleaned supragingivally with sterilized cotton pellets. A sterile absorbent paper point was inserted into the gingival crevice until resistance. The paper point was held in position for 30s. The samples were immediately put in Eppendorf tubes, transported to the laboratory and stored at $-80^{\circ} \mathrm{C}$. The collected samples were studied using the enzyme-linked immunosorbent assay (ELISA) technique of human resistin kit.

\section{Non-Surgical Periodontal Therapy:}

All patients in each groups were treated with nonsurgical periodontal therapy, which included the following: supragingival and subgingival scaling and root debridement were performed with an ultrasonic device, chlorhexidine mouthwash was prescribed twice daily for one week post periodontal therapy and oral hygiene instructions included teeth brushing using soft dental brush two times daily and suitable interdental aids once a day.

\section{Quantification of Human ResistinUsing ELISA Technique:}

Quantification of resistin in human samples was measured using the human resistin ELISA kit which is an in-vitro quantitative assay for detecting resistin peptide based on the principle of competitive enzyme immunoassay. The kit is suitable for testing a variety of sample types in vitro, such as serum, plasma, cell culture supernatants and urine and purchased from Sigma-Aldrich Co. US. The kit assayed resistin level in the sample, using a microplate which is pre-covered by anti-rabbit secondary antibody. Following a blocking step and incubation of the plate using anti-resistin antibody, both peptide standard (or targeted peptide) and biotinylated resistin peptide in samples reacts competitively with the resistin antibody. Uncompeted biotinylated resistin 
peptide then interacts with Streptavidin-horseradish peroxidase(SA-HRP), which develops a catalyzed color reaction. The strength of colorimetric signal is directly related to the amount of biotinylated peptide SA-HRP complex and inversely proportional to the quantity of resistin peptide in the samples. This is due to the competitive binding to resistin antibody between biotinylated resistin peptide and peptides in standard or samples. A standard curve of known resistin peptide concentrations can be recognized and the concentration of resistin peptide in the samples can be calculated accordingly. The resistin concentration in the specimens is then calculated by comparing the curve to the O.D. of the samples.

\section{Statistical Analysis:}

The results were presented as mean and standard deviation (SD) values. Data was analysed for normality using Kolmogorov-Smirnov test of normality. The results of Kolmogorov-Smirnov test demonstrated that data for CAL and PD were normally distributed (parametric data). Thus, one way analysis of variance (ANOVA) test was used for comparison purposes. Following that, Tukey's post hoc test was used when the difference was found to be considerable. Data for GI and PI were nonparametric, consequently Kruskall Wallis test was used to compare between groups and Friedman test was used to examine the effect of time. The significance level was set at $\mathrm{p} \leq 0.05$. Statistical analysis was done using SPSS 18.0 (The Statistical Package for Social Sciences, SPSS, Inc., Chicago, IL, USA) for Windows.

\section{RESULTS}

Table (1) showed the changes in the scores and measurements of PD and CAL from baseline, 1-month and 3-months after non-surgical periodontal therapy in each groups. In all groups, the highest mean value of each PI, GI, PD and CAL was recorded at baseline and a decrease was revealed in the following observation times.

Resistin ELISA analysis showed that the highest mean value of resistin level was recorded at baseline in group I $(0.040 \pm 0.017)$, group II $(0.083 \pm 0.027)$ and group III $(0.073 \pm 0.022)$ and statistically significant decrease was revealed in the following observation times $(\mathrm{P}=0.000)$ (Figure1). Tukey's post hoc test revealed a statistically significant difference between each 2 observation times in group I and III while revealed no statistically significant difference between each the 1 month and 3 months observation times in group II.

Table (1) Comparison of PI, GI, PD and CAL within the same group (effect by time), (One way ANOVA test).

\begin{tabular}{|l|c|c|c|c|c|c|c|c|c|}
\hline & \multicolumn{3}{|c|}{ Group I } & \multicolumn{3}{c|}{ Group II } & \multicolumn{3}{c|}{ Group III } \\
\cline { 2 - 10 } & Mean & SD & Pvalue & Mean & SD & P value & Mean & SD & P value \\
\hline PI & & & $0.00^{*}$ & & & $0.32^{\text {ns }}$ & & & $0.00^{*}$ \\
Base line & 1.90 & .74 & & 2.10 & .88 & & 2.60 & & \\
1 month & 1.30 & .48 & & 1.80 & .92 & & 1.60 & & \\
3 months & .40 & .52 & & 1.60 & 1.07 & & .40 & & \\
\hline GI & & & $0.00^{*}$ & & & $0.00^{*}$ & & & \\
Base line & 2.20 & .57 & & 2.30 & .67 & & 2.50 & .53 & \\
1 month & .80 & .39 & & 1.50 & .53 & & .30 & .48 & \\
3 months & .10 & .60 & & .60 & .32 & & .10 & .60 & \\
\hline PD & & & $0.15^{*}$ & & & $0.04 *$ & & & $0.35^{*}$ \\
Base line & 5.40 & .516 & & 5.40 & .483 & & 5.30 & .483 & \\
1 month & 4.30 & .675 & & 4.40 & .516 & & 4.30 & .483 & \\
3 month & 3.40 & .516 & & 3.80 & .789 & & 3.50 & .707 & \\
\hline CAL & & & $0.022^{*}$ & & & $0.039^{*}$ & & & $0.021^{*}$ \\
Base line & 6.50 & .527 & & 5.60 & .843 & & 6.40 & .516 & \\
1 month & 5.50 & .527 & & 5.20 & .422 & & 5.40 & .516 & \\
3 months & 4.60 & .516 & & 4.70 & .483 & & 4.80 & .789 & \\
\hline
\end{tabular}




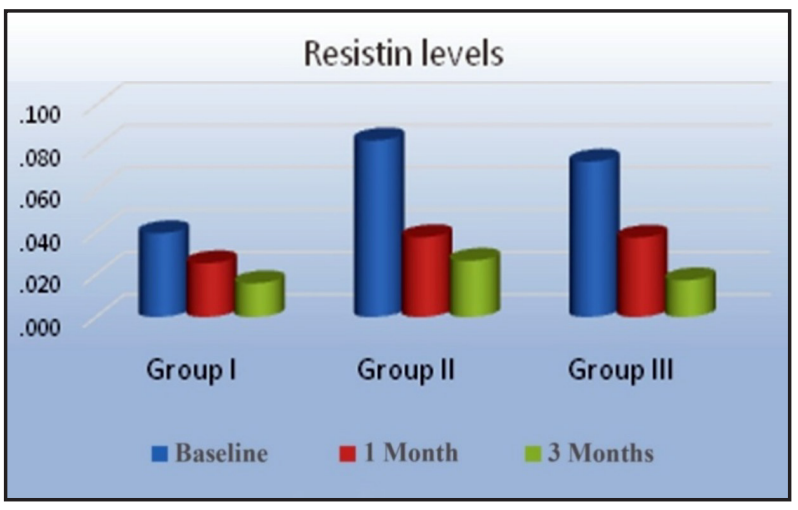

Figure (1) Column chart showing mean value of resistin levels in different groups.

At baseline, the highest mean value of resistin was recorded in group II $(0.083 \pm 0.027)$, whereas the lowest mean value was recorded in group I $(0.040 \pm 0.017)$, with no statistically significant difference between groups $(\mathrm{p}=0.30)$. At 1 month, the highest mean value was recorded in group II $(0.038 \pm 0.017)$, whereas the lowest mean value was recorded in group I $(0.025 \pm 0.009)$, with a statistically significant difference between groups $(\mathrm{p}=0.038)$. At 3 months, the highest mean value was recorded in group II $(0.027 \pm 0.008)$, whereas the lowest mean value was recorded in group I $(0.016 \pm 0.005)$, with a statistically significant between groups $(\mathrm{p}=0.011)$.

\section{DISCUSSION}

The relationship between diabetes mellitus and periodontitis has been a topic of study for years. At the mean time, there is powerful proof suggesting that the incidence and severity of periodontitis is affected by the absence or presence of diabetes mellitus, in addition to the extent to which the disease is controlled by patients. Furthermore, periodontitis could have a harmful effect on glycaemic control ${ }^{(20)}$.

Resistin, a putative adipocyte-derived signaling polypeptide, is named after its proposed function of resisting insulin. This cysteine-rich molecule belongs to the group of adipokines. Human studies suggest that very little resistin is expressed in adipocytes, it being largely expressed in neutrophils, macrophages, and monocytes. Human resistin also acts as a pro-inflammatory molecule and stimulates the synthesis and secretion of pro-inflammatory cytokines: TNF- $\alpha$, IL-6, interleukin-12IL-12 and monocyte chemoattractant protein (MCP)- $1^{(8)}$. So, it has been demonstrated that resistin levels in GCF can be considered as a biomarker for studying the severity of periodontitis and/or diabetic patients with periodontitis ${ }^{(12)}$.

Host modulation therapy seeks to improve conventional periodontal therapies by altering destructive aspects of the immune inflammatory host response, that results in reduction of periodontal breakdown and stabilization of periodontium. Such host modulation therapy involves usage of subantimicrobial dose of doxycycline (SDD $20 \mathrm{mg}$ twice daily) as an add-on to conventional nonsurgical periodontal therapy in the management of periodontitis $^{(14)}$. It is the single approved FDA host modulation agent to adjunctively treat periodontal disease without leading to antibiotic resistance by downregulation of $\mathrm{MMPs}^{(15)}$.

The results of the present study showed that all clinical parameters including PI, GI, PD, CAL in all groups, have decreased (improved) by time to reach the least mean value after 3 months of non-surgical periodontal therapy. These results are in agreement with several studies that showed either SRP alone or in conjunction with SDD could improve the clinical outcomes with systemically free periodontitis patients or T2DM periodontitis patients ${ }^{(21-23)}$.

In general, the present results after 3 months of treatment showed the greatest reduction of GI, PD and CAL in non-diabetic group compared to both diabetic groups. These findings were in accordance with several studies that reported that impaired glucose metabolism results in increasing GI, PD and $\mathrm{CAL}^{(24-25)}$.

However, at 3 month, group III showed more reduction in PI, GI and PD than group II with statistically significant difference. These results could be explained by the fact that SDD could enhance the 
results of a number of mechanical nonsurgical interventions by inhibition of MMPs which is involved in periodontal tissue destruction ${ }^{(26)}$.

Additionally, GCF levels of resistin significantly decreased by time in all groups after SRP to reach the lowest mean value at 3 month. Also, at 3 month, the present study found that group I showed the greatest reduction in GCF levels of resistin while group III showed more reduction in resistin levels compared with group II. This could be because of the increase in resistin secretion by the local inflammatory state in the periodontium, as well as a systemic inflammatory state owing to hyperglycemia ${ }^{(8)}$. In addition, this could be due to the probable tetracyclines' effect on expression, activation, and catalytic activity of MMPs. Moreover, these compounds could have an impact on other processes involved in the overall pathophysiology of multiple disease states, including regulation of glycosylation of connective tissue proteins and the release of inflammatory cytokines ${ }^{(27)}$.

In conclusion, periodontitis with T2DM patients recorded higher level of resistin in GCF than systemically free periodontitis patients. Non-surgical periodontal therapy resulted in a decrease in resistin level in all groups. In addition, SDD for short term was beneficial as an adjunct to non surgical periodontal therapy. Moreover, combined use of SRP with SDD resulted in more reduction in GI, PD and GCF resistin in periodontitisT2DMpatients compared to periodontitis patients with T2DM who didn't receive SDD.

\section{REFERENCS}

1. Papapanou PN, Sanz M, Buduneli N, Dietrich T, Feres M, Fine DH, et al. Periodontitis: Consensus report of workgroup 2 of the 2017 World Workshop on the Classification of Periodontal and Peri-Implant Diseases and Conditions, J periodontal, 2018; 89: 173-82.

2. Lalla E and Papapanou PN. Diabetes mellitus and periodontitis: a tale of two common interrelated diseases, Nat Rev Endocrinol, 2011; 7: 738 -48.
3. Salvi GE, Lawrence HP, Offenbacher S, Beck JD. Influence of risk factors on the pathogenesis of periodontitis, Periodontol, 2000; 14:173-201.

4. Wilton JM, Griffiths GS, Curtis MA, Maiden MF, Gillett IR, Wilson DT, et al. Detection of high risk groups and individuals for periodontal diseases. Systemic predisposition and markers of general health, J Clin Periodontol,1988; $15: 339-46$

5. Grossi SG and Genco RJ. Periodontal disease and diabetes mellitus: a two-way relationship, Ann Periodontol, 1998; 3:51-61.

6. Preshaw PM, Alba AL, Herrera D, Jepsen S, Konstantinidis A, Makrilakis K, et al. Periodontitis and diabetes: a twoway relationship, Diabetologia, 2012; 55:21-31.

7. Taylor JJ, Preshaw PM, Lalla E. A review of the evidence for pathogenic mechanisms that may link periodontitis and diabetes, J Clin Periodontol, 2013; 84: 113-34.

8. Gokhale NH, Acharya AB, Patil VS, Trivedi DJ, Setty S, Thakur SL. Resistin levels in gingival crevicular fluid of patients with chronic periodontitis and type 2 diabetes mellitus, , J Clin Periodontol, 2014; 85:610-7.

9. Banerjee RR and Lazar MA. Dimerization of resistin and resistin-like molecules is determined by a single cysteinem, J Biol Chem, 2001; 276: 25970 -3

10. Devanoorkar A,Kathariya R, Guttiganur N, Gopalakrishnan D, Bagchi P. Resistin: a potential biomarker for periodontitis influenced diabetes mellitus and diabetes induced periodontitis, Dis Markers; 2014: 930206.

11. Hiroshima Y, Bando M, Inagaki Y, Mihara C, Kataoka M, Murata $\mathrm{H}$, et al. Resistin in gingival crevicular fluid and induction of resistin release by Porphyromonas gingivalis lipopolysaccharide in human neutrophils, J Periodontal Res, 2012; 47:554-62.

12. Patel SP, Raju PA. Resistin in serum and gingival crevicular fluid as a marker of periodontal inflammation and its correlation with single-nucleotide polymorphism in human resistin gene at-420, Contemp Clin Dent, 2013; 4: 192-7.

13. Shinwari MS, Tanwir F, Hyder PR, Saeed MHB. Host modulation therapeutics in periodontics: role as an adjunctive periodontal therapy, J Coll Physicians Surg Pak, 2014; 24: 676-84.

14. Kaur $M$ and Krishan K. Subantimicrobial Dose Doxycycline in Treatment of Periodontitis, Periodontol, 2017; 3:1472-83. 
15. Silness $\mathbf{J}$ and Löe H. Periodontal disease in pregnancy II. Correlation between oral hygiene and periodontal condition. Acta Odontologica Scandinavica, 1964; 22: 121-35.

16. Löe H and Silness J. Periodontal disease in pregnancy I. Prevalence and severity, Acta Odontologica Scandinavical, 1963; 21: 533-51.

17. Ramfjord SP. Indices for prevalence and incidence of periodontal disease, J Periodontol, 1959; 30: 51-9.

18. Wiebe CB and Putnins EE. The periodontal disease classification system of the American Academy of Periodontologyan update, J Can Dent Assoc, 2000; 66:594-7.

19. Abramson JH. The Cornell Medical Index as an epidemiological tool, Am J Public Health Nations Health, 1966; 56: 287-98.

20. Rees TD. Periodontal management of the patient with diabetes mellitus, Periodontol 2000, 2000; 23: 63-72.

21. Choi DH, Moon IS, Choi BK, Paik JW, Kim YS, Choi $\mathrm{SH}$, et al. Effects of sub-antimicrobial dose doxycycline therapy on crevicular fluid MMP-8, and gingival tissue MMP-9, TIMP-1 and IL-6 levels in chronic periodontitis, J Periodontal Res, 2004: 39: 20-6.

22. Preshaw PM, Hefti AF, Jepsen S, Etienne D, Walker C, Bradshaw $\mathrm{MH}$, et al. Subantimicrobial dose doxycycline as adjunctive treatment for periodontitis: a review, J Clin Periodontol, 2004; 31: 697-707.

23. Michalowicz BS, Hyman L, Hou W, Oates TW, Reddy M, Paquette DW, et al. Factors associated with the clinical response to nonsurgical periodontal therapy in people with type 2 diabetes mellitus, J Am Dent Assoc, 20141; 145: 1227-39.

24. Tervonen C, Tellervo A, Matti K. Relation of diabetes control to periodontal pocketing and alveolar bone level, Oral Surg, Oral Med, Oral Pathol, 1986; 61:346-9.

25. Genco RJ, Grossi SG, Ho A, Nishimura F. Murayama, A proposed model linking inflammation to obesity, diabetes, and periodontal infections, J Periodontol, 2005; 76:2075-84.

26. Deo V, Gupta S, Bhongade ML, Jaiswal R. Evaluation of subantimicrobial dose doxycycline as an adjunct to scaling and root planing in chronic periodontitis patients with diabetes: a randomized, placebo-controlled clinical trial, J Contemp Dent Pract, 2010; 11:9-16.

27. Sasaki T, Ramamurthy NS, Golub LM. Tetracycline administration increases collagen synthesis in osteoblasts of streptozotocininduced diabetic rats: a quantitative autoradiographic study, Calcif Tissue Int, 1992: 50: 411-9. 